\title{
AN ABSTRACT FRAMEWORK FOR THE THEORY OF PROCESS OPTIMIZATION ${ }^{1}$
}

\author{
BY HUBERT HALKIN
}

Communicated by V. Klee, March 7, 1966

Introduction. Ten years ago the development of a maximum principle as a necessary condition for optimality of some control problems began a new era for optimization theory. Since that time different maximum principles have been proposed and proved for a great variety of optimization problems. All these maximum principles and their proofs have a similar structure. The aim of the present paper is to give this unique structure independently of the particular characteristics of any one of these problems.

The present paper is a further addition to the trend started in Gamkrelidze [1] and [2], Halkin [3] and [4], Neustadt [5].

1. Optimization problem. We are given a set $L$, a mapping $f=\left(f_{1}, f_{2}, \cdots, f_{k}\right)$ from $L$ into $E^{k}$ and an integer $m$ with $1 \leqq m \leqq k$. The problem is to find an $\hat{x} \in L$ which maximizes $f_{1}(\hat{x})$ subject to the constraints $f_{i}(\hat{x}) \geqq 0$ if $i=2,3, \cdots, m$ and $f_{i}(\hat{x})=0$ if $i=m+1, \cdots, k$.

2. Some assumptions. The set $L$ is a subset of a linear space $X$. There is a set $M \subset X$ which is an approximation of $L$ around $\hat{x}$ and a mapping $h=\left(h_{1}, \cdots, h_{k}\right): X \rightarrow E^{k}$ which is an approximation of $f$ around $\hat{x}$. We shall require that

(i) the set $M$ is convex and $\hat{x} \in M$.

(ii) the functionals $h_{i}$ are convex for $i=1, \cdots, m$ and linear-plusa-constant for $i=m+1, \cdots, k$.

(iii) for any set $S=\operatorname{co}\left\{\hat{x}, x_{1}, \cdots, x_{\imath}\right\} \subset M$ there is a mapping $\zeta: M \rightarrow L$ such that the mappings $f \circ \zeta$ and $h$ are continuous over $S$ (with respect to the usual finite dimensional topology on $S$ ) and "tangent at $\hat{x}$ over $S$ " which means that for any $\epsilon>0$ there is an $\eta \in(0,1]$ with the property that $|f(\zeta(x))-h(x)| \leqq \epsilon \delta$ if $\delta \in(0, \eta]$ and $x \in \operatorname{co}\left\{\hat{x}, \hat{x}+\delta\left(x_{1}-\hat{x}\right), \cdots, \hat{x}+\delta\left(x_{l}-\hat{x}\right)\right\}$.

3. Maximum principle. The purpose of the present paper is to prove that there exists real numbers $\lambda_{1}, \lambda_{2}, \cdots, \lambda_{k}$ such that

1 This research was supported by the Air Force Scientific Research Office of Aerospace Research, United States Air Force under AFOSR Grant 1039-66. 


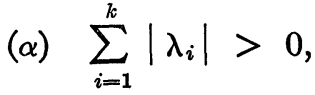

$$
\begin{aligned}
& \text { (B) } \quad \lambda_{1} \geqq 0 \quad \text { for } i=1,2, \cdots, m \text {, }
\end{aligned}
$$

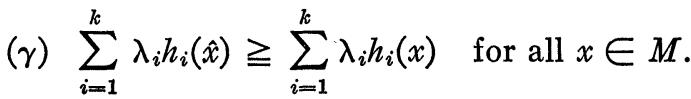

4. Proof of the maximum principle. There is no loss of generality by assuming that $\hat{x}=0$ and that $f(0)=0$. Let $K=\left\{\left(\alpha_{1}, \cdots, \alpha_{k}\right)\right.$ : $\left.\alpha_{i}>0, i=1, \cdots, m ; \alpha_{i}=0, i=m+1, \cdots, k\right\}$. We have $K \cap f(L)$ $=\varnothing$. We want to prove that $K$ and $h(M)$ are separated. We shall assume that $K$ and $h(M)$ are not separated and show that this leads to $K \cap f(L) \neq \varnothing$. If the sets $h(M)$ and $K$ are not separated then, Step I, there exists a set $S=\operatorname{co}\left\{0, x_{1}, \cdots, x_{l}\right\} \subset M$ such that

(i) $h(S)$ and $K$ are not separated,

(ii) $l=k-m+1$,

(iii) $h_{j}\left(x_{i}\right)>0$ for $j=1, \cdots, m$ and $i=1, \cdots, l$.

Let $S^{0}=S \sim\{0\}$. Then, Step II, there exists a $\sigma>0$ such that $h\left(S^{0}\right) \subset\left\{\rho\left(\alpha_{1}, \cdots, \alpha_{k}\right): \rho \in(0,1], \sigma \leqq \alpha_{i} \leqq 1 / \sigma, i=1, \cdots, m ;-1 / \sigma\right.$ $\left.\leqq \alpha_{i} \leqq 1 / \sigma, i=m+1, \cdots, k\right\}$. For every $\delta \in(0,1]$ let $S_{\delta}^{0}=\{\delta x$ : $x \in S \sim\{0\}\}$. Then, Step III, there exists a $\beta \in(0,1]$ such that $f_{i}(\zeta(x))>0$ if $i=1, \cdots, m$ and $x \in S_{\beta}^{0}$ where $\zeta$ is the mapping from $S$ in to $L$ given by the definition of $M$. Then, Step IV, $f(\zeta(S)) \cap K \neq \varnothing$ which implies $f(L) \cap K \neq \varnothing$. This concludes the proof of the Maximum Principle. Steps I, II and III correspond to elementary properties of convex sets and convex functions in a finite dimensional Euclidean space. Step IV is a consequence of Brouwer fixed point theorem.

\section{REFERENCES}

1. R. V. Gamkrelidze, On the theory of the first variation, Dokl. Akad. Nauk SSSR 161 (1965), 23-26 = Soviet Math. 6 (1965), 345-348.

2. - On some extremal problems in the theory of differential equations with applications to the theory of optimal control, SIAM J. Control, 3 (1965), 106-128.

3. H. Halkin, Finite convexity in infinite dimensional spaces, Proceedings of the Colloquium on Convexity, Copenhagen, 1965 (to appear).

4. - On the necessary condition for optimal control of nonlinear systems, J. Analyse Math., 12 (1964), 1-82.

5. L. W. Neustadt, Optimal control problems as extremal problems in a Banach space, Proceedings of the Symposium on System Theory, Polytechnic Institute of Brooklyn, 1965 (to appear).

University of California, la Jolla 